\title{
EXTRAÇÃO E EXPORTAÇÃO DE NUTRIENTES POR VARIEDADES DE CANA-DE-AÇÚCAR CULTIVADAS SOB IRRIGAÇÃO PLENA ${ }^{(1)}$
}

\author{
Emídio Cantídio Almeida de Oliveira ${ }^{(2)}$, Fernando José Freire ${ }^{(3)}$, \\ Ruthanna Isabelle de Oliveira ${ }^{(4)}$, Maria Betânia Galvão dos Santos \\ Freire $^{(3)}$, Djalma Euzébio Simões Neto ${ }^{(5)} \&$ Silas Alves Monteiro da \\ Silva ${ }^{(4)}$
}

\section{RESUMO}

A pesquisa tecnológica para suporte do setor sucroalcooleiro nacional mostra que são esporádicos os trabalhos desenvolvidos com cana-de-açúcar irrigada envolvendo a exigência nutricional. Nesse contexto, objetivou-se quantificar, durante o ciclo de cana-planta de 11 variedades de cana-de-açúcar (SP79-1011, RB813804, RB863129, RB872552, RB943365, RB72454, RB763710, SP78-4764, SP813250, RB867515 e RB92579) cultivadas sob irrigação plena, a capacidade de extração e exportação de $\mathrm{N}, \mathrm{P}, \mathrm{K}, \mathrm{Ca}$ e Mg, bem como a exigência nutricional para produção de uma tonelada de colmo por hectare (TCH). A pesquisa foi realizada em campo, no município de Carpina, PE, durante a safra agrícola 2006/2007. O delineamento experimental empregado foi de blocos casualizados, com 11 tratamentos e quatro repetições. A extração e exportação de nutrientes, assim como a exigência nutricional, foram avaliadas aos 360 dias após o plantio (DAP) na parte aérea das plantas. A extração de nutrientes na parte aérea da cana-planta apresentou, em média, valores de 179, 25, 325, 226 e $87 \mathrm{~kg} \mathrm{ha}^{-1} \mathrm{de} \mathrm{N}, \mathrm{P}, \mathrm{K}, \mathrm{Ca}$ e Mg, respectivamente, o que proporcionou a seguinte ordem decrescente de extração: $\mathrm{K}>\mathrm{Ca}>\mathrm{N}>\mathrm{Mg}>\mathrm{P}$. A exportação média de N, $\mathrm{P}, \mathrm{K}, \mathrm{Ca}$ e Mg pelo colmo das variedades irrigadas foi de $92 ; 15 ; 188 ; 187$; e $66 \mathrm{~kg} \mathrm{ha}^{-1}$, correspondendo, respectivamente, a 51, 60, 58, 83 e $76 \%$ de todo o nutriente extraído na parte aérea da cana-planta, com destaque para as variedades RB92579 e SP81-3250 para o N, RB813804, RB863129, RB872552, RB763710, RB92579, SP78-4764, SP81-3250 e SP79-1011 para o P, SP79-1011, SP81-

\footnotetext{
(1) Parte da Dissertação de Mestrado do primeiro autor apresentada ao Programa de Pós-Graduação em Ciência do Solo, Universidade Federal Rural de Pernambuco - UFRPE. Recebido para publicação em setembro de 2009 e aprovado em junho de 2010.

(2) Doutorando em Ciência do Solo e Nutrição de Plantas da Escola Superior de Agricultura "Luis de Queiroz" - ESALQ/USP, Caixa postal 9, CEP 13418-900 Piracicaba (SP). Bolsista FAPESP. E-mail: ecaoliveira@cena.usp.br

(3) Professor do Departamento de Agronomia, Universidade Federal Rural de Pernambuco - UFRPE. Rua Dom Manuel de Medeiros s/n, Dois Irmãos, CEP 52171-900 Recife (PE). E-mails: f.freire@depa.ufrpe.br; betania@depa.ufrpe.br

(4) Estagiários do Centro de Pesquisa de Solos, UFRPE. Bolsista CNPq. E-mail: ruth_yisa@hotmail.com; silasalves@hotmail.com

(5) Pesquisador e Coordenador da Estação Experimental de Cana-de-açúcar de Carpina - EECAC/UFRPE. Rua Ângela Cristina C. Pessoa de Luna s/n, CEP 55810-700 Carpina (PE). E-mail: desn@oi.com.br
} 
3250, RB813804, RB872552 e RB763710 para o K, RB92579 e RB863129 para o Ca e RB92579 para o Mg. Para produção de uma TCH, foram exigidos pelas variedades durante o ciclo de cana-planta valores médios de 0,$91 ; 0,13 ; 1,71 ; 1,18$; e $0,44 \mathrm{~kg}$ de $\mathrm{N}$, $\mathrm{P}, \mathrm{K}, \mathrm{Ca}$ e Mg, respectivamente.

Termos de indexação: cana-planta, exigência nutricional, Saccharum spp.

\title{
SUMMARY: NUTRIENT EXTRACTION AND EXPORT BY FULLY IRRIGATED SUGARCANE VARIETIES
}

\begin{abstract}
A review of technology research supporting the Brazilian sugaralcohol sector shows that only sporadic studies with irrigated sugarcane have been developed to investigate nutritional requirements. In this context, the objective of this study was to quantify the capacity of extraction and export of $N, P, K, C a$, and $M g$ as well as the nutritional requirement for the production of one ton of stalk per hectare (TCH) in the plant cane cycle of 11 sugarcane varieties (SP79-1011, RB813804, RB863129, RB872552, RB943365, RB72454, RB763710, SP78-4764, SP81$3250, R B 867515$, and $R B 92579)$ grown under full irrigation. The research was conducted under field conditions in the municipality of Carpina, PE, in the 2006/2007 growing season. The experiment was arranged in a randomized block design with 11 treatments and four replications. Nutrient extraction and export as well as nutritional requirements of the shoot components of the plants were evaluated 360 days after planting. The mean nutrient accumulation in shoots was 179, 25, 325, 226, and $87 \mathrm{~kg} \mathrm{ha}^{-1}$ of $\mathrm{N}, \mathrm{P}, \mathrm{K}, \mathrm{Ca}$, and $\mathrm{Mg}$, respectively. The average export of $N, P, K, C a$, and $M g$ via stalks of the irrigated varieties was $92,15,188,187$, and $66 \mathrm{~kg} \mathrm{ha} \mathrm{h}^{-1}$, corresponding, respectively, to $51,60,58,83$, and $76 \%$ of the whole nutrient amount taken up by the cane plant. The extracted nutrient amounts were highest by the following varieties: RB92579 and SP81-3250 for N; RB813804, RB863129, RB872552, RB763710, RB92579 SP78-4764, SP81-3250 and SP79-1011 for P; SP791011, SP81-3250, RB813804, RB872552 and RB763710 for $K$; RB92579 and RB863129 for $\mathrm{Ca}$; and $\mathrm{RB} 92579$ for $\mathrm{Mg}$, for which the export values in stalks were highest. For the production of one TCH, the varieties needed $0.91,0.13,1.71,1.18$, and $0.44 \mathrm{~kg}$ of $\mathrm{N}, \mathrm{P}, \mathrm{K}, \mathrm{Ca}$, and $M g$, respectively.
\end{abstract}

Index term: sugar cane, nutritional requirement, Saccharum spp.

\section{INTRODUÇÃO}

Nas regiões produtoras de cana-de-açúcar do Brasil, o volume de chuvas irregularmente distribuído, adicionalmente à diversidade dos solos cultivados com essa cultura, que apresentam características químicas e físicas variadas, tem justificado o investimento do setor sucroalcooleiro na seleção de variedades adaptadas a diferentes condições edafoclimáticas, bem como na adoção da irrigação nos canaviais, o que leva a seleção de variedades de cana-de-açúcar a não se restringir apenas ao potencial produtivo, mas também à capacidade da planta em transformar de forma eficiente o nutriente absorvido em biomassa.

O conhecimento da exigência nutricional da canade-açúcar torna-se fundamental para o estudo da adubação, indicando a quantidade de nutrientes que deve ser fornecida (Coleti et al., 2006). Contudo, o total de nutrientes extraídos do solo pelas plantas varia entre variedades, ciclo de cultivo, manejo do solo e disponibilidade de nutrientes (Ceotto \& Castelli, 2002), tornando necessário novos estudos que identifiquem a interação desses fatores, para correta recomendação de variedades e métodos de cultivo nos diferentes ambientes de produção.

Estudo realizado por Franco et al. (2007) em Latossolo Vermelho mostrou que o acúmulo de nutrientes na parte aérea da variedade SP81-3250 na colheita de cana-planta apresentou a seguinte ordem decrescente: $\mathrm{K}>\mathrm{N}>\mathrm{Ca}>\mathrm{S}>\mathrm{Mg}>\mathrm{P}$. Por outro lado, Coleti et al. (2006), estudando as variedades RB835486 e SP81-3250, em dois Argissolos Vermelhos, constataram, para cana-planta, a seguinte ordem de extração de nutrientes: $\mathrm{K}>\mathrm{N}>\mathrm{S}>\mathrm{P}>\mathrm{Mg}>\mathrm{Ca}$, e na cana-soca: $\mathrm{K}>\mathrm{N}>\mathrm{P}>\mathrm{Mg}>\mathrm{S}>\mathrm{Ca}$. Em variedade mais antiga e menos produtiva, como a CB41-76, cultivada em dois Latossolos Vermelhos, como também em Argissolo Vermelho, a exigência pelos macronutrientes não seguiu o mesmo padrão das variedades atualmente plantadas, obtendo-se na canaplanta e cana-soca a ordem de extração de: $\mathrm{K}>\mathrm{N}>$ $\mathrm{Ca}>\mathrm{Mg}>\mathrm{S}>\mathrm{P}$ (Orlando Filho et al., 1980). 
Pesquisas que atuam na identificação do potencial de extração e exportação de nutrientes em novas variedades e sob condições de irrigação são esporádicas e pouco consistentes. Atualmente as informações referem-se às variedades Co 419 e CB 41-76, que não são mais cultivadas (Orlando Filho et al., 1980; Sobral \& Weber, 1983), ou a estudos conduzidos em condições de sequeiro, que não demonstraram o máximo potencial produtivo e de extração de nutrientes pelas variedades, devido à limitação das condições edafoclimáticas de cada ambiente de produção. Nessas condições de cultivo, resultados de pesquisa em diferentes variedades e classes de solo revelaram que a exigência nutricional para produção de uma tonelada de colmo por hectare (TCH) variou de 0,92 a $1,80 \mathrm{~kg}$ de N, 0,09 a 0,17 de P, 0,63 a 3,2 de K, 0,11 a 0,56 de $\mathrm{Ca}, 0,13$ a 0,48 de $\mathrm{Mg}$ e 0,15 a 0,28 de $\mathrm{S}$ (Orlando Filho et al., 1980; Coleti et al., 2006; Tasso Junior et al., 2007; Franco et al., 2008b).

A identificação da demanda por nutrientes relacionada à produção de colmos nas variedades atualmente utilizadas nos canaviais brasileiros é indispensável para obtenção de alta produtividade, assim como para recomendar corretamente variedades para condições de baixo suprimento de nutrientes pelo solo ou para situações que permitam o máximo desenvolvimento da cultura quando combinadas com o uso da irrigação (Karthikeyan et al., 2003; Rakkiyappan et al., 2005).

O objetivo deste trabalho foi quantificar, durante o ciclo de cana-planta de 11 variedades de cana-deaçúcar (SP79-1011, RB813804, RB863129, RB872552, RB943365, RB72454, RB763710, SP78-4764, SP813250, RB867515 e RB92579) cultivadas sob irrigação plena, a capacidade de extração e exportação de N, P, $\mathrm{K}$, Ca e Mg, bem como a exigência nutricional para produção de uma tonelada de colmo por hectare.

\section{MATERIAL E MÉTODOS}

A pesquisa foi conduzida em campo localizado na área agrícola da Estação Experimental de Cana-deAçúcar de Carpina (EECAC), Unidade de Pesquisa da Universidade Federal Rural de Pernambuco (UFRPE), situado no município de Carpina, com latitude de $7^{\circ} 51$ ' 133 " S, longitude de $35^{\circ} 14$ ' 102 " W e altitude de $180 \mathrm{~m}$. A pesquisa foi desenvolvida durante o ciclo de cana-planta, durante o período de outubro de 2006 a outubro de 2007. Nesse período, foram quantificadas temperaturas médias superiores a $25^{\circ} \mathrm{C}$ e precipitação pluvial anual de $1.181 \mathrm{~mm}$, distribuída irregularmente, proporcionando déficit hídrico acentuado em determinados períodos de crescimento da cultura (Figura 1).

O solo da área experimental foi classificado como Argissolo Amarelo distrófico abrúptico (Embrapa, 2006) textura franco-arenosa/franco-argiloarenosa, cuja caracterização física e química foi realizada em amostras de solo coletadas nas camadas de 0,0-0,2, 0,2-0,4 e 0,4-0,6 m (Quadro 1). O preparo do solo consistiu de gradagem para destruição dos resíduos culturais, previamente dessecados com herbicida, e incorporação do calcário, seguida da abertura dos sulcos de plantio. Utilizou-se calcário na dose de $465 \mathrm{~kg} \mathrm{ha}^{-1}$, calculado pelo método da neutralização do $\mathrm{Al}$ trocável ou elevação dos teores de $\mathrm{Ca}$ e $\mathrm{Mg}$ trocáveis, considerando-se $3,0 \mathrm{cmol}_{\mathrm{c}} \mathrm{dm}^{-3}$ como nível crítico de $\mathrm{Ca}+\mathrm{Mg}$ (IPA, 2008).

A adubação do solo foi baseada no Manual de Recomendação de Adubação para o Estado de Pernambuco (IPA, 2008), conforme os resultados da análise do solo (Quadro 1), aplicando-se no fundo do sulco de plantio $30 \mathrm{~kg} \mathrm{ha}^{-1}$ de N, $120 \mathrm{~kg} \mathrm{ha}^{-1} \mathrm{de}_{2} \mathrm{O}_{5}$ e $70 \mathrm{~kg} \mathrm{ha}^{-1}$ de $\mathrm{K}_{2} \mathrm{O}$. O plantio foi realizado manualmente, sendo os colmos de cana-de-açúcar com

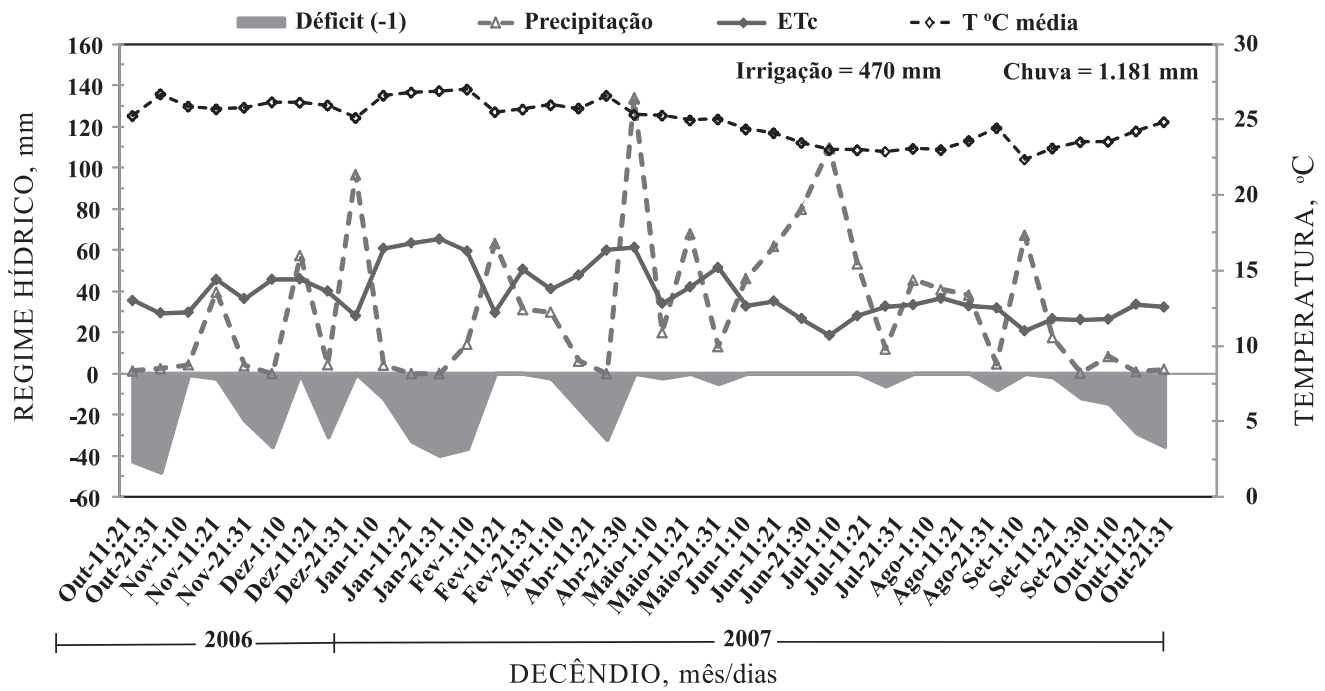

Figura 1. Balanço hídrico e temperatura média do ar durante a condução do experimento. 
três gemas por rebolo (tolete), e distribuídos nos sulcos de plantio de modo que atingissem 18 gemas por metro. Aos 90 dias após o plantio (DAP), realizou-se a adubação de cobertura, aplicando-se $50 \mathrm{~kg} \mathrm{ha}^{-1}$ de N e $50 \mathrm{~kg} \mathrm{ha}^{-1}$ de $\mathrm{K}_{2} \mathrm{O}$ nas linhas da cana, seguida de incorporação manual.

Durante o ciclo de cana-planta (2006/2007) foram adicionados, por irrigação, $470 \mathrm{~mm}$ de água, o que totalizou $1.651 \mathrm{~mm}$ de água efetiva. A irrigação foi manejada de forma plena, com aplicação de uma lâmina de água necessária para suprir a soma diária da evapotranspiração da cultura (ETc). Calculou-se uma lâmina útil para irrigar a cultura até a profundidade de $0,4 \mathrm{~m}$ nos três primeiros meses e 0,6 $\mathrm{m}$ até os 300 DAP (Quadro 1). Para isso, levaram-se em consideração os resultados da capacidade de campo (CC) e do ponto de murcha permanente (PMP) do solo até a respectiva profundidade, bem como a precipitação e a eficiência (75 \%) do sistema de irrigação utilizado. Nos 60 dias que antecederam a colheita não foi adicionada água por irrigação, objetivando reduzir o crescimento vegetativo e estimular a maturação fisiológica; entretanto, verificaram-se precipitações de $96 \mathrm{~mm}$ para esse período (Figura 1).

A ETc foi calculada segundo a equação: ETc = ECA x Kp x Kc, em que ETc é a evapotranspiração da cultura (mm); ECA, a evaporação do tanque Classe A (mm); Kp, o coeficiente do tanque Classe A; e Kc, o coeficiente de cultura. Os valores de coeficientes de cultura seguiram os diversos estádios de desenvolvimento da cana-de-açúcar, sendo utilizados os valores de 0,$55 ; 0,8 ; 1,0 ; 1,25 ; 1,0 ;$ e 0,6 , respectivamente na germinação, perfilhamento, estabelecimento do estande, crescimento elevado ou elongação do colmo, senescência das folhas e maturação (Gomes, 1994; Inman-Bamber \& McGlinchey, 2003; Bernardo et al., 2005). Na reposição da lâmina, utilizou-se o sistema de aspersão convencional do tipo canhão móvel com bocal de quatro polegadas de diâmetro, com vazão (Q) de $54 \mathrm{~m}^{3} \mathrm{~h}^{-1}$ sob pressão de $40 \mathrm{~m}$ de coluna de água (mca).

O experimento foi conduzido avaliando-se 11 variedades de cana-de-açúcar, sendo cinco de maturação precoce (SP79-1011, RB813804, RB863129, RB872552 e RB943365) e seis de maturação média a tardia (RB72454, RB763710, SP78-4764, SP81-3250, RB867515 e RB92579), em delineamento experimental de blocos ao acaso, com quatro repetições, perfazendo o total de 44 parcelas experimentais, que constaram de cinco linhas de cana-de-açúcar, com $10 \mathrm{~m}$ de comprimento, espaçadas de 1,10 m. A área útil da parcela abrangeu os três sulcos centrais com $8 \mathrm{~m}$ de comprimento.

Aos 360 DAP foi amostrada aleatoriamente a parte aérea de oito plantas na área útil de cada parcela experimental. As amostras foram separadas nos componentes ponteiro, folha e colmo, sendo o ponteiro constituído do cartucho e da folha +1 (primeiro colarinho visível); para a componente folha (folha + bainha), consideraram-se as folhas secas e verdes a partir da folha +1 ; e, após a retirada do ponteiro e das folhas, o restante foi considerado colmo. Após a amostragem das plantas destinadas às estimativas de extração e exportação dos nutrientes, determinouse a produção de colmos, pesando-se ainda no campo, com auxílio de um dinamômetro, os colmos contidos na área útil de cada parcela experimental, calculandose em seguida a produção por hectare $\left(\mathrm{t} \mathrm{ha}^{-1}\right)$.

Os ponteiros, folhas e colmos, após separados, foram pesados, determinando-se a massa de matéria fresca total. As amostras de material vegetal fresco foram trituradas em forrageira industrial e, em seguida, coletaram-se subamostras úmidas. No laboratório, as subamostras foram submetidas à

\section{Quadro 1. Caracterização química e física do solo em três profundidades}

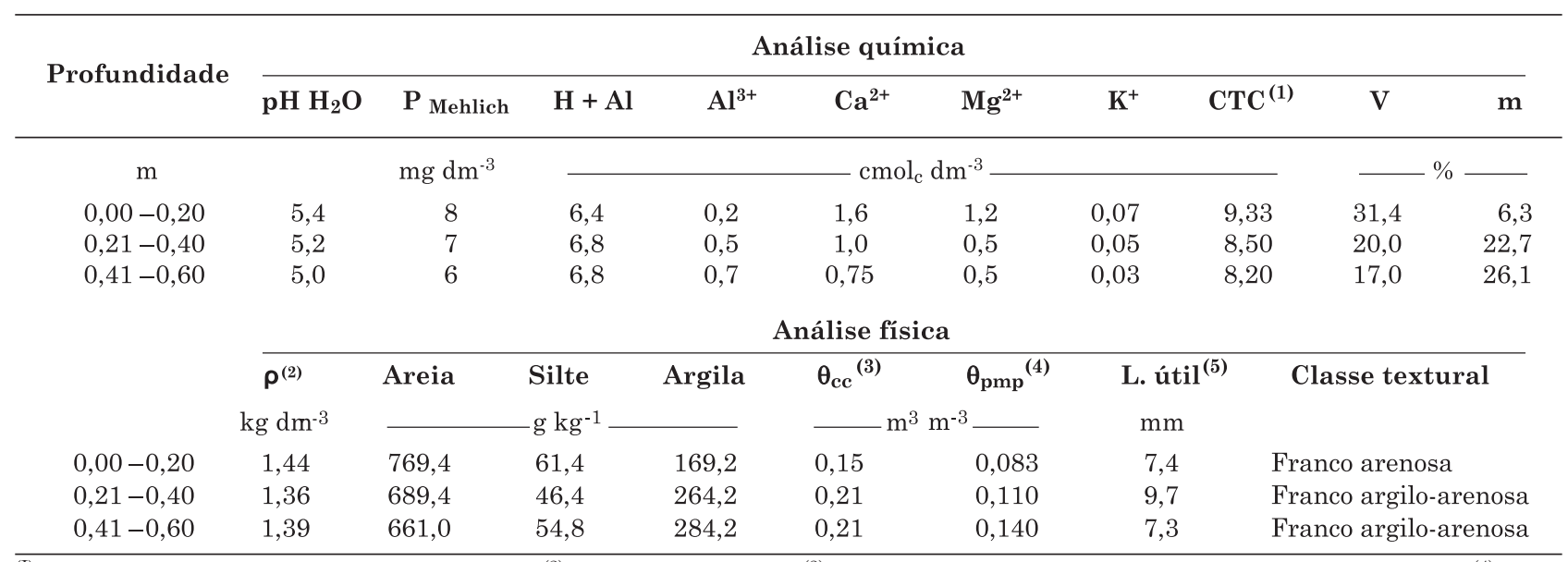

(I) Capacidade de troca de cátions potencial. ${ }^{(2)}$ Densidade do solo. ${ }^{(3)}$ Umidade do solo na capacidade de campo a 0,1 MPa. ${ }^{(4)}$ Umidade do solo no ponto de murcha permanente a $1,5 \mathrm{MPa} .{ }^{(5)}$ Lâmina de água útil calculada a partir de $50 \%$ da lâmina total. 
secagem em estufa de circulação forçada de ar a $65{ }^{\circ} \mathrm{C}$ até massa constante e novamente pesadas para determinação da umidade do material. Após obtenção da massa seca, as subamostras foram passadas em moinho tipo Wiley, para em seguida se determinar a concentração de $\mathrm{N}$, que foi obtida por digestão sulfúrica e determinada por titulação. As concentrações de $\mathrm{P}$, $\mathrm{K}, \mathrm{Ca}$ e $\mathrm{Mg}$ foram obtidas por digestão nitroperclórica, determinando-se $\mathrm{P}$ por colorimetria, $\mathrm{K}$ por fotometria de chama e Ca e Mg por espectrofotometria de absorção atômica (Malavolta et al., 1997).

A extração dos nutrientes pela parte aérea das variedades foi calculada somando-se o produto da massa de matéria seca pela concentração do nutriente contido em cada componente (ponteiros, folhas e colmo). Os nutrientes alocados no colmo foram considerados como o total de nutriente exportado. A exigência nutricional foi estimada dividindo-se a extração total de nutrientes na parte aérea pela produção de colmos de cada variedade.

Os dados foram submetidos à análise da variância em delineamento de blocos casualizados, utilizandose o teste F a $1 \%$. Para comparar a extração, exportação e a exigência por nutrientes das 11 variedades, utilizou-se o teste de Scott-Knott a $5 \%$.

\section{RESULTADOS E DISCUSSÃO}

As variedades diferiram nas quantidades extraídas de nutrientes e apresentaram a seguinte ordem decrescente de extração: $\mathrm{K}>\mathrm{Ca}>\mathrm{N}>\mathrm{Mg}>\mathrm{P}$ (Quadro 2), com exceção da variedade SP81-3250, que extraiu mais $\mathrm{N}$ do que $\mathrm{Ca}$, corroborando os resultados encontrados por Franco et al. (2007) e Coleti et al.
(2006) nessa mesma variedade, conduzida sem irrigação, durante o ciclo de cana-planta. Por outro lado, as variedades RB867515 e SP78-4764 apresentaram extrações de $\mathrm{N}$ próximas à de $\mathrm{Ca}$ (Quadro 2), confirmando o que foi observado por Coale et al. (1993) na variedade CL61-620 quando cultivada em Histosols na região da Flórida/EUA. A menor extração de $\mathrm{N}$ pela maior parte das variedades pode ser atribuída à dose utilizada no ensaio, uma vez que as elevadas produtividades de cultivos irrigados aumenta na demanda por N. Adicionalmente, o solo em que se cultivaram as variedades tinha textura média com tendência arenosa em superfície (Quadro 1), favorecendo perdas por lixiviação, principalmente pelo manejo utilizado.

Os dados médios de exportação de N, P, K, Ca e $\mathrm{Mg}$ das variedades revelaram que 51, 60, 58, 83 e $76 \%$, respectivamente, dos nutrientes absorvidos foram exportados pelo colmo da cana-de-açúcar durante o ciclo de cana-planta (Quadro 2). É provável que a baixa mobilidade do Ca no floema (Maathuis, 2009) não permita sua redistribuição na planta, fazendo com que se acumule no colmo, elevando, consequentemente, sua exportação na colheita.

Analisando especificamente a extração e exportação de cada nutriente em função das diferentes variedades, constatou-se que a extração de $\mathrm{N}$ diferiu estatisticamente entre as variedades $(p<0,01)$, verificando-se que a RB92579 apresentou a maior extração (260 kg ha-1 de N) (Quadro 2). Com extrações intermediárias, identificaram-se estatisticamente quatro grupos de variedades: RB867515 e SP81-3250, que acumularam na parte aérea 237 e $241 \mathrm{~kg} \mathrm{ha}^{-1} \mathrm{de}$ $\mathrm{N}$, respectivamente; SP78-4764 e RB863129, que extraíram 201 e $196 \mathrm{~kg} \mathrm{ha}^{-1}$, respectivamente; RB943365 e RB763710, que acumularam 177 e

Quadro 2. Extração e exportação de nitrogênio, fósforo, potássio, cálcio e magnésio em diferentes variedades de cana-de-açúcar conduzidas sob manejo de irrigação plena

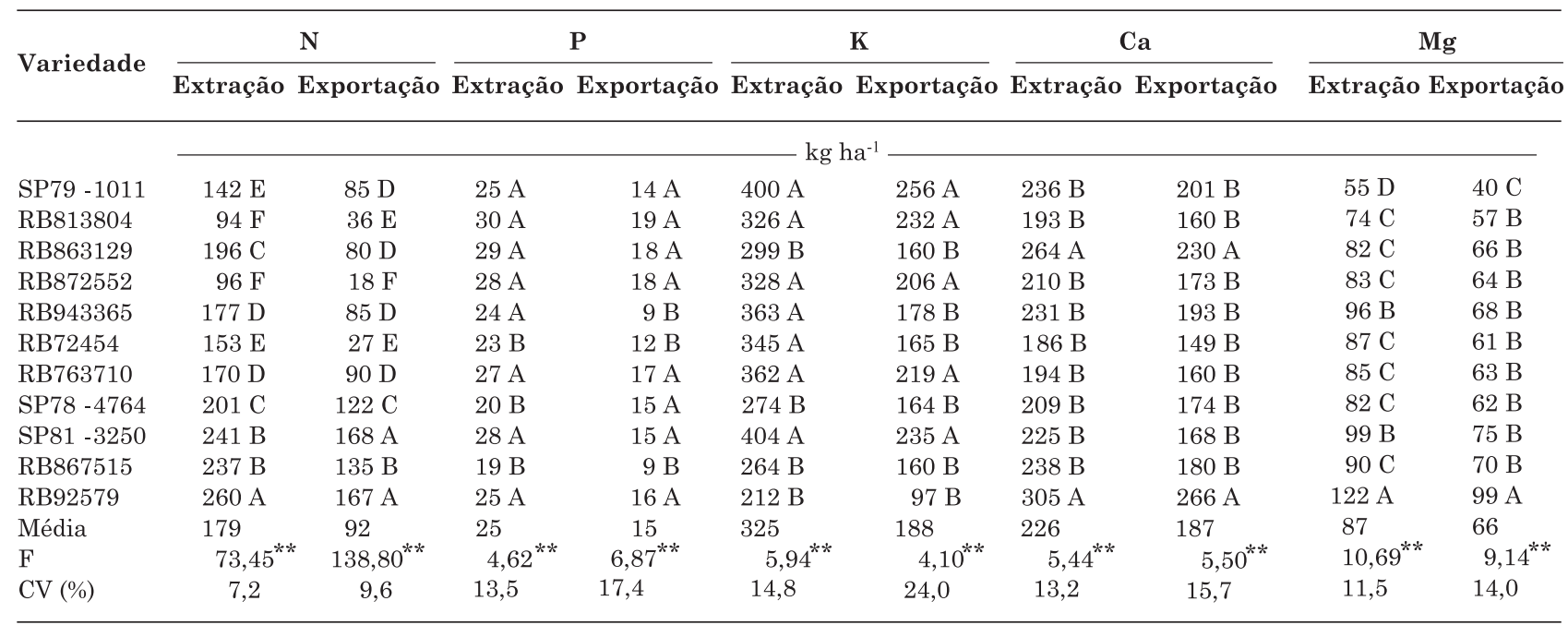

Médias seguidas pela mesma letra, na coluna, não diferem entre si pelo teste de Scott-Knott a $5 \%$; ${ }^{* * *}$ Significativo a $1 \%$ pelo teste F. 
170 kg ha ${ }^{-1}$ de N, respectivamente; e RB72454 e SP791011, que extraíram 153 e $142 \mathrm{~kg} \mathrm{ha}^{-1}$ de $\mathrm{N}$, respectivamente. As menores extrações de $\mathrm{N}$ foram observadas em RB872552 e RB813804, que apresentaram, respectivamente, remoções de 96 e $94 \mathrm{~kg} \mathrm{ha}^{-1}$ de N (Quadro 2). Oliveira et al. (2002), avaliando extração de N nas variedades RB867515 e RB72454 sob irrigação de salvação, constataram acúmulos na parte aérea de 271 e $198 \mathrm{~kg} \mathrm{ha}^{-1}$ de N, respectivamente. Por outro lado, quando não irrigadas, as variedades RB867515 (Silva et al., 2007a) e RB72454 (Barbosa et al., 2002) apresentaram extrações de 149 e $134 \mathrm{~kg} \mathrm{ha}^{-1}$ de $\mathrm{N}$, respectivamente. Também em manejo de sequeiro, as variedades SP813250 (Coleti et al., 2006; Franco et al., 2008a) e RB92579 (Silva et al., 2007a) apresentaram extrações de 185 e $148 \mathrm{~kg} \mathrm{ha}^{-1}$ de $\mathrm{N}$, respectivamente.

Para o $\mathrm{N}$ alocado no colmo, que corresponde efetivamente ao $\mathrm{N}$ exportado, constatou-se nas variedades RB72454 e RB872552 que, apesar de diferirem estatisticamente nas quantidades exportadas (Quadro 2), cujas exportações foram, respectivamente, de 27 e $18 \mathrm{~kg} \mathrm{ha}^{-1}$ de $\mathrm{N}$, elas representaram apenas 18 e $19 \%$, respectivamente, do N acumulado na parte aérea da planta. Entretanto, nas variedades SP81-3250 e RB92579, que não diferiram estatisticamente, as exportações foram de 168 e $167 \mathrm{~kg} \mathrm{ha}^{-1}$, respectivamente, o que correspondeu a 70 e $64 \%$, respectivamente, de todo o $\mathrm{N}$ extraído (Quadro 2).

Quanto às demais variedades, RB867515 e SP784764 exportaram quantidades diferentes entre si (135 e $122 \mathrm{~kg} \mathrm{ha}^{-1}$ de $\mathrm{N}$, respectivamente) e, também, diferiram das outras variedades. Com exportações de $\mathrm{N}$ intermediárias, identificou-se o grupo das variedades RB763710, RB943365, SP79-1011 e RB863129. Com menores exportações de N apresentaram-se as variedades RB813804 e RB72454. A relação entre quantidade exportada e extraída de $\mathrm{N}$ pelas variedades mostrou variação percentual de 18 a $70 \%$. Genericamente, variedades de comportamento mais extrator de $\mathrm{N}$ também foram grandes exportadoras. Variações nas exportações entre $16 \mathrm{e}$ $60 \%$ de todo o $\mathrm{N}$ acumulado na parte aérea foram encontradas na literatura (Coale et al., 1993; Wood et al., 1996; Korndorffer et al., 1997; Gava et al., 2001; Rakkiyappan et al., 2007), corroborando os resultados obtidos neste trabalho.

Os dados de extração de $\mathrm{P}$ pelas variedades mostraram remoção média de $25 \mathrm{~kg} \mathrm{ha}^{-1}$ de $\mathrm{P}$ e diferiram estatisticamente entre elas (Quadro 2), sendo observado que nas variedades RB813804, RB863129, RB872552, SP81-3250, RB763710, RB92579, SP79-1011 e RB943365 não houve diferença na extração de P. Entretanto, as menores extrações ocorreram nas variedades RB72454, SP78-4764 e RB867515, que não diferiram entre si e apresentaram valores de extração de 23,20 e $19 \mathrm{~kg} \mathrm{ha}^{-1}$ de $\mathrm{P}$, respectivamente (Quadro 2). A extração de $\mathrm{P}$ na variedade RB867515 foi inferior ao valor observado por Oliveira et al. (2002) quando cultivou essa variedade sob irrigação em Latossolo Vermelho no Estado de Minas Gerais e constatou remoção de $29 \mathrm{~kg} \mathrm{ha}^{-1}$ de $\mathrm{P}$, porém próximo ao valor obtido na mesma variedade por Silva et al. (2007b) quando manejou o cultivo em regime de sequeiro nos tabuleiros costeiros de Alagoas, constatando extração de $21 \mathrm{~kg} \mathrm{ha}^{-1}$ de $\mathrm{P}$. Constatou-se, também, que a extração de $\mathrm{P}$ obtida por esses mesmos autores na variedade $\mathrm{RB} 92579$ (23 $\mathrm{kg} \mathrm{ha}^{-1}$ de $\left.\mathrm{P}\right)$ foi próxima à obtida neste trabalho (25 kg ha-1 de P) (Quadro 2). As maiores remoções de $\mathrm{P}$ pelas variedades utilizadas foram próximas às obtidas por outros autores em diferentes condições de cultivo e com uso de outras variedades (Chiranjivi Rao \& Thangavelu, 2004; Rakkiyappan et al., 2005; Franco et al., 2008a); contudo, acúmulos de apenas $5 \mathrm{~kg} \mathrm{ha}^{-1}$ de $\mathrm{P}$ foram obtidos por Prado et al. (2002) na parte aérea da variedade SP80-1842 cultivada em Latossolo Vermelho Amarelo no Estado de São Paulo.

Os dados de acúmulos de $\mathrm{P}$ no colmo mostraram que aos 360 DAP as exportações diferiram estatisticamente entre as variedades e variaram de 14 a $19 \mathrm{~kg} \mathrm{ha}^{-1}$ de $\mathrm{P}$ nas variedades que mais exportaram e de 9 a $12 \mathrm{~kg} \mathrm{ha}^{-1}$ de $\mathrm{P}$ nas que menos acumularam esse nutriente no colmo (Quadro 2). A exportação nas variedades que mais acumularam $\mathrm{P}$ no colmo foi responsável, em média, por $63 \%$ da extração de P, destacando-se a SP78-4764, que exportou $75 \%$ do que extraiu. Nas variedades que menos exportaram, as perdas de $\mathrm{P}$ pelo colmo foram, em média, de $46 \%$, sendo apenas de $37 \%$ na variedade RB943365. Silva et al. (2007b) relataram extrações de $\mathrm{P}$ entre 36 e $51 \%$ na colheita de quatro variedades cultivadas em Cururipe-AL. Em outras condições edafoclimáticas, Chiranjivi Rao \& Thangavelu (2004) e Rakkiyappan et al. (2007) observaram que as exportações de $\mathrm{P}$ corresponderam entre 36 e $75 \%$ da extração por diferentes variedades cultivadas na India. Exportações de $\mathrm{P}$ ainda maiores foram encontradas nas variedades RB72454 (Barbosa et al., 2002) e SP813250 (Coteli et al., 2006) sob cultivo de sequeiro, correspondendo, em média, a $79 \%$ da extração na cana-planta.

No tocante à extração de $\mathrm{K}$, constatou-se que as variedades SP81-3250, SP79-1011, RB943365, RB763710, RB72454, RB872552 e RB813804 acumularam as maiores quantidades desse nutriente e não diferiram estatisticamente entre si (Quadro 2). As maiores extrações pela parte aérea foram verificadas nas variedades SP81-3250 e SP79-1011, com valores de 404 e $400 \mathrm{~kg} \mathrm{ha}^{-1}$ de K, respectivamente (Quadro 2). Franco et al. (2008b) também constataram na variedade SP81-3250 alta capacidade de acumular K na parte aérea, com extrações médias de $390 \mathrm{~kg} \mathrm{ha}{ }^{-1}$ de K. Quantidades menores de K e estatisticamente semelhantes foram extraídas pelas variedades RB863129, SP78-4764, RB867515 e RB92579, sendo de apenas $212 \mathrm{~kg} \mathrm{ha}^{-1}$ de $\mathrm{K}$ na 
RB92579 (Quadro 2), apesar da elevada capacidade produtiva desta variedade (Quadro 3). Em média, as variedades extraíram $325 \mathrm{~kg} \mathrm{ha}^{-1}$ de K (Quadro 2), que corresponderam a valores inferiores aos obtidos por Oliveira et al. (2002), os quais relataram extrações de $334 \mathrm{~kg} \mathrm{ha}^{-1}$ de Kem sete variedades de cana-de-açúcar irrigadas. Coale et al. (1993) também relataram extrações de $343 \mathrm{~kg} \mathrm{ha}^{-1}$ de K em condições de sequeiro para dois ciclos de cultivo na Flórida/EUA. Silva et al. (2008), quando conduziram as variedades RB92579 e RB867515 em condições de sequeiro, verificaram que as extrações de K foram superiores a SP79-1011, diferindo dos resultados obtidos neste trabalho, evidenciando comportamento diferente na extração de $\mathrm{K}$, dependendo do manejo de cultivo.

No que se refere ao acúmulo de K no colmo, constatou-se que as variedades mais extratoras foram as mais exportadoras, com exceção de RB72454 e RB943365 (Quadro 2). As variedades que menos exportaram K foram RB943365, RB72454, SP78-4764, RB863129, RB867515 e RB92579, que apresentaram acúmulo no colmo de 178, 165, 164, 160, 160 e $97 \mathrm{~kg} \mathrm{ha}^{-1}$ de K, respectivamente (Quadro 2). As exportações foram responsáveis, em média, por $58 \%$ da extração de $\mathrm{K}$, considerando todas as variedades, destacando-se a variedade RB813804, que exportou mais de $70 \%$ do $\mathrm{K}$ acumulado na parte aérea, enquanto a variedade RB92579 exportou apenas $46 \%$ do que absorveu. Silva et al. (2008), trabalhando com esta mesma variedade, relataram exportações que representaram $43 \%$ do que foi extraído. Coale et al. (1993) constataram exportações de $53 \%$ do K acumulado na parte aérea, corroborando os dados médios obtidos neste trabalho.

A análise dos dados de extração de Ca constatou que as variedades RB92579 e RB863129 apresentaram as maiores extrações, com 305 e $264 \mathrm{~kg} \mathrm{ha}^{-1}$ de Ca, respectivamente, bem como as maiores exportações, com acúmulos no colmo de 266 e $230 \mathrm{~kg} \mathrm{ha}^{-1} \mathrm{de} \mathrm{Ca}$, respectivamente (Quadro 2). Essas variedades não diferiram estatisticamente nas quantidades extraídas e exportadas, porém diferiram de todas as demais variedades, que extraíram entre 186 e $238 \mathrm{~kg} \mathrm{ha}^{-1}$ de Ca e exportaram entre 201 e $193 \mathrm{~kg} \mathrm{ha}^{-1}$ de Ca (Quadro 2), não se observando diferença entre elas nas quantidades de $\mathrm{Ca}$ avaliadas. Em média, o acúmulo de Ca na parte aérea das variedades foi de $226 \mathrm{~kg} \mathrm{ha}^{-1}$ de Ca valor esse superior às extrações encontradas por outros pesquisadores em regime de manejo irrigado (Oliveira et al., 2002) e em sequeiro (Coale et al., 1993; Prado et al., 2002; Coleti et al., 2006).

A exportação de Ca pelo colmo correspondeu, em média, a $83 \%$ de todo o nutriente extraído pela planta, valor esse inferior aos descritos por Coale et al. (1993), que relataram uma relação exportação/extração de $94 \%$. Em outros trabalhos que avaliaram a capacidade de extração e exportação de diferentes variedades de cana-de-açúcar, o total exportado variou de 52 a $76 \%$ (Thangavelu \& Chiranjivi Rao, 2004; Coleti et al., 2006).
A variedade RB92579 apresentou as maiores remoções de $\mathrm{Mg}$ do solo, sendo diferente estatisticamente das outras variedades (Quadro 2). Com remoções intermediárias, identificaram-se dois grupos de variedade: o das SP81-3250 e RB943365, que extraíram 99 e $96 \mathrm{~kg} \mathrm{ha}^{-1}$ de $\mathrm{Mg}$, respectivamente; e o das RB867515, RB72454, RB763710, RB872552, RB863129, SP78-4764 e RB813804, que extraíram 90, $87,85,83,82,82$ e $74 \mathrm{~kg} \mathrm{ha}^{-1} \mathrm{de} \mathrm{Mg}$, respectivamente. Com a menor remoção de $\mathrm{Mg}$, a variedade SP79-1011 extraiu apenas $55 \mathrm{~kg} \mathrm{ha}^{-1} \mathrm{de} \mathrm{Mg}$. As extrações de Mg encontradas neste trabalho superaram os resultados obtidos por outros pesquisadores, tanto em condições de manejo irrigado (Oliveira et al., 2002) quanto de sequeiro (Silva et al., 2009).

Em relação às exportações de $\mathrm{Mg}$, foram verificados na variedade RB92579 os maiores valores, apresentando perdas pelo colmo de $99 \mathrm{~kg} \mathrm{ha}^{-1}$, que equivaleram a $81 \%$ do nutriente acumulado na parte aérea, contrastando com a menor exportação obtida pela variedade SP79-1011, a qual apresentou valores de $40 \mathrm{~kg} \mathrm{ha}^{-1}$, que correspondeu a $72 \%$ de todo o $\mathrm{Mg}$ extraído pela planta (Quadro 2). Para as demais, os acúmulos de $\mathrm{Mg}$ no colmo não mostraram diferença estatística, constatando-se exportações médias de $65 \mathrm{~kg} \mathrm{ha}^{-1}$ (Quadro 2). Resultados de outros trabalhos mostraram que a relação exportação/extração variou de $58 \%$ (Thangavelu \& Chiranjivi Rao, 2004) a $90 \%$ (Coale et al., 1993) em condições de manejo não irrigado.

O cultivo das variedades sob manejo de irrigação plena proporcionou rendimento médio de colmo da ordem de $195 \mathrm{t} \mathrm{ha}^{-1}$ (Quadro 3), destacando-se as variedades RB92579, SP81-3250 e RB763710, que apresentaram, respectivamente, produtividades médias de 256, 232 e $212 \mathrm{t} \mathrm{ha}^{-1}$ de colmos. Os altos rendimentos apresentados pelas variedades foram responsáveis por maiores remoções de nutrientes do solo, superando, de modo geral, as extrações encontradas em variedades conduzidas em manejo sem irrigação por outros pesquisadores (Coale et al., 1993; Barbosa et al., 2002; Prado et al., 2002; Thagavelu \& Chiranjivi Rao, 2004; Coleti et al., 2006; Silva et al., 2007a, b; Franco et al., 2008a,b; Silva et al., 2008).

Assim, para responder à irrigação, torna-se cada vez mais necessária a seleção de variedades que sejam eficientes na utilização de nutrientes e que possuam a capacidade de adaptar-se aos diversos ambientes de produção nos quais a cana-de-açúcar irrigada encontrase inserida.

Avaliando as relações entre extrações de nutrientes e produção, constatou-se que RB867515 e SP784764 exigiram mais $\mathrm{N}\left(1,27\right.$ e $1,12 \mathrm{~kg} \mathrm{t}^{-1} \mathrm{de} \mathrm{N}$, respectivamente) do que as variedades SP81-3250, RB92579 e $\operatorname{RB763710}\left(1,04,1,02\right.$ e $0,80 \mathrm{~kg} \mathrm{t}^{-1}$ de N, respectivamente), porém foram menos produtivas (Quadro 3), evidenciando menor eficiência na utilização do $\mathrm{N}$ absorvido. As menores exigências nutricionais de $\mathrm{N}$ foram obtidas nas variedades RB872552 e RB813804, 
Quadro 3. Produção de colmos por hectare (TCH) e extração de nitrogênio, fósforo, potássio, cálcio e magnésio em diferentes variedades de cana-de-açúcar conduzidas sob manejo de irrigação plena, necessários para produção de uma tonelada de colmo

\begin{tabular}{|c|c|c|c|c|c|c|}
\hline Variedade & TCH & $\mathbf{N}$ & $\mathbf{P}$ & $\mathbf{K}$ & $\mathrm{Ca}$ & Mg \\
\hline & $\mathrm{t} \mathrm{ha}^{-1}$ & & 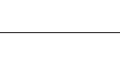 & $-\mathrm{kg} \mathrm{t}^{-1}$ & 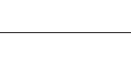 & - \\
\hline SP79 - 1011 & $155 \mathrm{~B}$ & $0,92 \mathrm{~B}$ & $0,16 \mathrm{~A}$ & $2,58 \mathrm{~A}$ & $1,52 \mathrm{~A}$ & $0,35 \mathrm{~B}$ \\
\hline RB813804 & $177 \mathrm{~B}$ & $0,53 \mathrm{C}$ & $0,17 \mathrm{~A}$ & $1,84 \mathrm{~B}$ & $1,09 \mathrm{~B}$ & $0,42 \mathrm{~A}$ \\
\hline RB863129 & $181 \mathrm{~B}$ & $1,08 \mathrm{~B}$ & $0,16 \mathrm{~A}$ & $1,65 \mathrm{C}$ & $1,46 \mathrm{~A}$ & $0,45 \mathrm{~A}$ \\
\hline RB872552 & $176 \mathrm{~B}$ & $0,55 \mathrm{C}$ & $0,16 \mathrm{~A}$ & $1,86 \mathrm{~B}$ & $1,19 \mathrm{~A}$ & $0,47 \mathrm{~A}$ \\
\hline RB943365 & $193 \mathrm{~B}$ & $0,92 \mathrm{~B}$ & $0,12 \mathrm{~B}$ & $1,88 \mathrm{~B}$ & $1,20 \mathrm{~A}$ & $0,50 \mathrm{~A}$ \\
\hline RB72454 & 192 B & $0,80 \mathrm{~B}$ & $0,12 \mathrm{~B}$ & $1,80 \mathrm{~B}$ & $0,97 \mathrm{~B}$ & $0,45 \mathrm{~A}$ \\
\hline RB763710 & $212 \mathrm{~A}$ & $0,80 \mathrm{~B}$ & $0,13 \mathrm{~B}$ & $1,71 \mathrm{~B}$ & $0,92 \mathrm{~B}$ & $0,40 \mathrm{~A}$ \\
\hline SP78-4764 & $179 \mathrm{~B}$ & $1,12 \mathrm{~A}$ & $0,11 \mathrm{~B}$ & $1,53 \mathrm{C}$ & $1,17 \mathrm{~A}$ & $0,46 \mathrm{~A}$ \\
\hline SP81-3250 & $232 \mathrm{~A}$ & $1,04 \mathrm{~B}$ & $0,12 \mathrm{~B}$ & $1,74 \mathrm{~B}$ & $0,97 \mathrm{~B}$ & $0,43 \mathrm{~A}$ \\
\hline RB867515 & $186 \mathrm{~B}$ & $1,27 \mathrm{~A}$ & $0,10 \mathrm{~B}$ & $1,42 \mathrm{C}$ & $1,28 \mathrm{~A}$ & $0,48 \mathrm{~A}$ \\
\hline RB92579 & $256 \mathrm{~A}$ & $1,02 \mathrm{~B}$ & $0,10 \mathrm{~B}$ & $0,83 \mathrm{C}$ & $1,19 \mathrm{~A}$ & $0,48 \mathrm{~A}$ \\
\hline Média & 195 & 0,91 & 0,13 & 1,71 & 1,18 & 0,44 \\
\hline $\mathrm{F}$ & $5,53^{* *}$ & $9,05^{*}$ & $7,11^{* *}$ & $6,83^{* *}$ & $2,99^{*}$ & $9,63^{*}$ \\
\hline CV (\%) & 12,5 & 17,1 & 13,5 & 18,2 & 17,7 & 20,0 \\
\hline
\end{tabular}

Médias seguidas pela mesma letra, na coluna, não diferem entre si pelo teste de Scott-Knott a 5 \%; ${ }^{* * * *}$ Significativo, respectivamente, a 5 e $1 \%$ pelo teste $\mathrm{F}$.

que necessitaram de apenas 0,55 e $0,53 \mathrm{~kg} \mathrm{t}^{-1}$ de $\mathrm{N}$, respectivamente, o que representou cerca de 2,4 vezes menos do que exigiu a RB867515.

Para P, constatou-se que as variedades RB813804, RB722552, RB863129 e SP79-1011 revelaram-se menos eficientes, demandando 0,17;0,16;0,16; e 0,16 kg t-1 de $\mathrm{P}$ para atingirem menores produtividades, quando comparadas com as variedades SP81-3250, RB92579 e RB763710 (Quadro 3), que não diferiram na demanda por P.

Dentre as variedades menos exigentes em K, destacaram-se RB92579, RB867515, SP78-4764 e RB863129, que apresentaram demandas de 0,$83 ; 1,42$; 1,53; e 1,65 kg t-1 de K, respectivamente (Quadro 3). Saliente-se que a variedade RB92579, apesar da baixa exigência em K, apresentou elevada produtividade. Por outro lado, a variedade SP79-1011 mostrou-se a mais exigente em $K$, requerendo $2,58 \mathrm{~kg} \mathrm{t}^{-1}$ de $\mathrm{K}$, porém não converteu essa exigência em produtividade, sobretudo quando comparada à variedade RB92579. Elevada exigência de K pela variedade SP81-3250 foi constatada por Franco et al. (2008b) em dois solos do Estado de São Paulo. Esses autores afirmaram serem necessários 3,54 kg de K para produção de uma TCH. Por sua vez, Silva et al. (2008) não observaram elevadas exigências de K na variedade SP79-1011 para a mesma produção de colmos.

Para o Ca, verificou-se que as variedades RB763710, RB72454, SP81-3250 e RB813804 mostraram-se pouco exigentes na utilização desse nutriente, sendo necessários 0,$92 ; 0,97 ; 0,97$; e $1,09 \mathrm{~kg} \mathrm{t}^{-1}$ de $\mathrm{Ca}$ (Quadro 3). A elevada produtividade das variedades SP81-3250 e RB763710 e a pouca exigência em Ca credenciam essas variedades como eficientes na utilização desse nutriente. Com relação ao $\mathrm{Mg}$, sua exigência nutricional diferiu entre as variedades, e a SP79-1011 foi a menos exigente, requerendo apenas $0,35 \mathrm{~kg} \mathrm{t}^{-1} \mathrm{de} \mathrm{Mg}$, credenciando-a para utilização em ambientes mais restritivos à disponibilidade desse nutriente. A exigência de $\mathrm{Ca}$ e Mg observada nas variedades irrigadas foi superior à verificada em variedades conduzidas em regime de sequeiro, quando se constataram requerimentos entre 0,11 e $0,65 \mathrm{~kg} \mathrm{t}^{-1}$ de Ca e entre 0,13 e $0,37 \mathrm{~kg} \mathrm{t}^{-1} \mathrm{de}$ $\mathrm{Mg}$ (Thangavelu \& Chiranjivi Rao, 2004; Coleti et al., 2006). Franco et al. (2008b), avaliando a extração de nutrientes pela variedade SP81-3250 em dois Latossolos Vermelhos, também observaram variações nas exigências de $\mathrm{Ca}$ e $\mathrm{Mg}$, evidenciando que a demanda por nutriente é dependente não apenas da variedade utilizada, mas também de sua interação com o ambiente de cultivo.

O uso de variedades mais eficientes na utilização de $\mathrm{Ca}$ e $\mathrm{Mg}$ pode ser uma indicação para alocação dessas variedades em solos relativamente ácidos e pobres nesses nutrientes, ou seja, em ambientes de baixa a média fertilidade natural. Entretanto, o potencial de resposta à irrigação por esse tipo de variedade foi limitado, quando comparado com variedades mais produtivas e menos eficientes (Quadro 3). Assim, a recomendação de variedades para áreas irrigadas concomitantemente com solos de baixa fertilidade natural deve levar em consideração, prioritariamente, a capacidade delas em manter elevadas produções durante a longevidade do canavial, devido ao alto investimento inicial atribuído ao sistema de irrigação.

Além do incremento na produção de colmos, a prática da irrigação levou as variedades irrigadas a tornarem-se mais exigentes quando comparadas com 
as extrações obtidas por variedades conduzidas sem irrigação (Silva et al., 2007a,b; Tasso Junior et al., 2007; Franco et al., 2008a,b; Silva et al., 2008), sendo necessários, em média, 0,$91 ; 0,13 ; 1,71 ; 1,18$; e $0,44 \mathrm{~kg} \mathrm{t}^{-1}$ de $\mathrm{N}, \mathrm{P}, \mathrm{K}, \mathrm{Ca}$ e $\mathrm{Mg}$, respectivamente (Quadro 3).

\section{CONCLUSÕES}

1. A extração de nutrientes e alocação na parte aérea da cana-planta apresentaram, em média, valores de 179, 25, 325, 226 e $87 \mathrm{~kg} \mathrm{ha}^{-1}$ de N, P, K, Ca e Mg, respectivamente, o que proporcionou a seguinte ordem decrescente de extração: $\mathrm{K}>\mathrm{Ca}>\mathrm{N}>\mathrm{Mg}>\mathrm{P}$.

2. A exportação média de $\mathrm{N}, \mathrm{P}, \mathrm{K}, \mathrm{Ca}$ e $\mathrm{Mg}$ pelo colmo das variedades irrigadas foi de 92, 15, 188, 187 e $66 \mathrm{~kg} \mathrm{ha}^{-1}$, correspondendo, respectivamente, a 51, $60,58,83$ e $76 \%$ de todo o nutriente extraído na parte aérea da cana-planta.

3. As variedades RB92579 e SP81-3250 foram as que mais exportaram N; RB813804, RB863129, RB872552, RB763710, RB92579, SP78-4764, SP813250 e SP79-1011, o P; SP79-1011, SP81-3250, RB813804, RB872552, RB763710, K; RB92579 e RB863129, o Ca; e RB92579; o Mg.

4. Para produção de uma TCH, foram exigidos pelas variedades, durante o ciclo de cana-planta, valores médios de 0,$91 ; 0,13 ; 1,71 ; 1,18 ;$ e 0,44 kg de N, P, K, $\mathrm{Ca}$ e $\mathrm{Mg}$, respectivamente.

\section{AGRADECIMENTOS}

À Estação Experimental de Cana-de-Açúcar de Carpina e ao Programa de Pós-Graduação em Ciência do Solo da UFRPE, pela disponibilidade de estrutura física e pessoal e pelo auxílio na execução da pesquisa e na realização das análises laboratoriais.

\section{LITERATURA CITADA}

BARBOSA, M.H.P.; OLIVEIRA, M.W.; SILVEIRA, L.C.I.; DAMASCENO, C.M. \& MENDES, L.C. Acúmulo e alocação de nutrientes pela RB72454 no ciclo da cana-planta. In: CONGRESSO NACIONAL DA SOCIEDADE DOS TÉCNICOS ACCUCAREIROS E ALCOOLEIROS DO BRASIL, 8., Recife, 2002. Anais... Recife, Sociedade dos Técnicos Açucareiros e Alcooleiros do Brasil, 2002. p.264267.

BERNARDO, S.; SOARES, A.A. \& MANTOVANI, E.C. Manual de irrigação. 7.ed. Viçosa, MG, Universidade Federal de Viçosa, 2005. 611p.

CHIRANJIVI RAO, K. \& THANGAVELU, S. Uptake of nitrogen, phosphorus and potassium in above ground parts by intergeneric hybrids. Sugar Tec., 6:15-23, 2004.
COALE, F.J.; SANCHEZ, C.A.; IZUNO, F.T. \& BOTTCHER, A.B. Nutrient accumulation and removal by sugar cane grown on everglades histosols. Agron. J., 85:310-315, 1993.

CEOTTO, E. \& CASTELLI, F. Radiation-use efficiency in fluecured tobacco (Nicotiana tabaccum L.): Response to nitrogen supply, climatic variability and sink limitation. Field Crops Res., 74:117-130, 2002.

COLETI, J.T.; CASAGRANDE, J.C.; STUPIELLO, J.J.; RIBEIRO, L.D. \& OLIVEIRA, G.R. Remoção de macronutrientes pela cana-planta e cana-soca, em Argissolos, variedades RB83486 e SP81-3250. STAB, 24:32-36, 2006.

EMPRESA BRASILEIRA DE PESQUISA AGROPECÚARIA EMBRAPA. Centro Nacional de Pesquisa de Solos. Sistema brasileiro de classificação de solos. 3.ed. Rio de Janeiro, 2006. 306p.

EMPRESA PERNAMBUCANA DE PESQUISA AGROPECUÁRIA - IPA. Recomendações de adubação para o estado de Pernambuco. 2.ed . Recife, 1998. 198p.

FRANCO, H.C.J.; TRIVELIN, P.C.O.; OTTO, R.; FARONI, C.E.; VITTI, A.C.; SARTORI; R.H. \& CANTARELLA, H. Acúmulo de nitrogênio, fósforo e potássio, durante o ciclo de cana-planta, relacionados à adubação nitrogenada. In: CONGRESSO NACIONAL DA SOCIEDADE DOS TÉCNICOS AÇUCAREIROS E ALCOOLEIROS DO BRASIL, 8., Maceió, 2008. Anais... Maceió, Sociedade dos Técnicos Açucareiros e Alcooleiros do Brasil, 2008a. p.228234.

FRANCO, H.C.J.; TRIVELIN, P.C.O.; VITTI, A.C.; OTTO, R.; FARONI, C.E.; SARTORI, R.H. \& TRIVELIN, M.O. Acúmulo de nutrientes pela cana-de-açúcar. STAB, 26:47$51,2008 b$

FRANCO, H.C.J.; BOLONGA, I.R.; FARONI, C.E.; VITTI, A.C. \& TRIVELIN, P.C.O. Acúmulo de macronutrientes em cana-de-açúcar em função da adubação nitrogenada e dos resíduos culturais incorporados ao solo no plantio. Bragantia, 66:669-674, 2007.

GAVA, J.C.; TRIVELIN, P.C.O.; OLIVEIRA, M.W. \& PENATTI, C.P. Crescimento e acúmulo de nitrogênio em cana-deaçúcar cultivada em solo coberto com palha. Pesq. Agropec. Bras., 36:1.347-1.354, 2001.

GOMES, H.P. Engenharia de irrigação: Hidráulica dos sistemas pressurizados, aspersão e gotejamento. João Pessoa, EDUFPB, 1994. 344p.

INMAN-BAMBER, N.G. \& MCGLINCHEY, M.G. Crop coefficients and water use estimates for sugarcane based on long-term Bowen ratio energy balance measurements. Field Crops Res., 83:125-138, 2003.

KARTHIKEYAN, P.K.; BANSAL, S.K.; DHAKSHINAMOORTHY, M. \& KRISHNASAMY, R. Effect of potassium fertilization on the cane yield, juice quality and economics sugar cane grown on farmers' fields of Tamil Nadu. Fert. News, 48:31$38,2003$.

KORNDORFFER, G.H.; VALLE, M.R.; MARTINS, M. \& TRIVELIN, P.C.O. Avaliação do nitrogênio da uréia pela cana-planta. R. Bras. Ci. Solo, 21:223-226, 1997. 
MALAVOLTA, E.; VITTI, G.C. \& OLIVEIRA, S.A. Avaliação do estado nutricional das plantas. Princípios e aplicações. 2.ed. Piracicaba, Potafos, 1997. 201p.

MAATHUIS, F.J.M. Physiological functions of mineral macronutrients. Curr. Opin. Plant. Biol., 12:150-158, 2009.

OLIVEIRA, M.W.; MENDES, L.C.; BARBOSA, M.H.P.; VITTI, A.C. \& FARIA, R.O. Avaliação de sete variedades de cana-de-açúcar sob irrigação complementar. In: REUNIÃO BRASILEIRA DE FERTILIDADE DO SOLO E NUTRIÇÃO DE PLANTAS. 15., Rio de Janeiro, 2002. Anais... Rio de Janeiro, Sociedade Brasileira de Ciência do Solo, 2002. CD-ROM.

ORLANDO FILHO. J.; HAAG, H.P. \& ZAMBELLO JUNIOR, E. Crescimento e absorção de macronutrientes pela canade-açúcar, variedade CB41-76, em função da idade, em solos do estado de São Paulo. São Paulo, IAA, 1980. 128p. (Boletim Técnico PLANALSUCAR, 2).

PRADO, R.M.; FERNANDES, F.M. \& NATALE, W. Calcário e escória de siderurgia avaliados por análise foliar, acúmulo e exportação de macronutrientes em cana-de-açúcar. Sci. Agric., 59:129-135, 2002.

RAKKIYAPPAN, P.; THANGAVELU, S.; BHAGYALAKSHMI, K.V. \& RADHAMANI, R. Uptake of nitrogen, phosphorus and potassium by soma promising mid late maturing sugarcane clones. Sugar Tec., 9:27-27, 2007.

RAKKIYAPPAN, P.; THANGAVELU, S.; SHANTHI, R.M.; ALARMELU, S. \& RADHAMANI, R. Uptake of major nutrients in above ground parts of promising mid late maturing sugarcane clones. Sugar Tec., 7:67-70, 2005.

SILVA, L.C.; MOURA FILHO, G.; SILVA, V.T.; MOURA, A.B.; RODRIGUES, W.A.R.; CERQUEIRA, D.C.O.; FERREIRA, L.C.R.; CARNAÚBA, P.J.P. \& ALMEIDA, C.A.B. Remoção de magnésio em sete cultivares de cana-de-açúcar (Saccharum spp) na região de Coruripe-AL. In: CONGRESSO BRASILEIRO DE CIÊNCIA DO SOLO, 32., Fortaleza, 2009. Anais... Fortaleza, Sociedade Brasileira de Ciência do Solo, 2009. CD-ROM.
SILVA, L.C.; MOURA FILHO, G.; SILVA, V.T.; FERREIRA, L.C.R.; CARNAÚBA, P.J.P.; ALMEIDA, C.A.B.; SOUZA, J.L.; COSTA, J.V.T. \& SANTOS, V.R. Acúmulo e eficiência de potássio em sete cultivares de cana-de-açúcar na Região de Cururipe-AL. In: CONGRESSO NACIONAL DA SOCIEDADE DOS TÉCNICOS AÇUCAREIROS E ALCOOLEIROS DO BRASIL, 9., Maceió, 2008. Anais... Maceió, Sociedade dos Técnicos Açucareiros e Alcooleiros do Brasil, 2008. p.244-248.

SILVA, L.C.; MOURA FILHO, G.; SILVA, V.T.; FERREIRA, L.C.R.; CARNAÚBA, P.J. P.; ALMEIDA, C.A.B. \& ALBUQUERQUER, A.W. Remoção de nitrogênio em quatro variedades de cana-de-açúcar (Saccharum spp) na região de Coruripe-AL. In: CONGRESSO BRASILEIRO DE CIÊNCIA DO SOLO, 31., Gramado, 2007. Anais. Gramado, Sociedade Brasileira de Ciência do Solo, 2007a. CD-ROM.

SILVA, L.C.; MOURA FILHO, G.; SILVA, V.T.; FERREIRA, L.C.R.; CARNAÚBA, P.J. P.; ALMEIDA, C.A.B. \& COSTA, J.P.V. Acúmulo de $\mathrm{P}$ em quatro variedades de cana-deaçúcar (Saccharum spp) na região de Coruripe-AL. In: CONGRESSO BRASILEIRO DE CIÊNCIA DO SOLO, 31., Gramado, 2007, Anais. Gramado, Sociedade Brasileira de Ciência do Solo, 2007b. CD-ROM.

SOBRAL, A.F. \& WEBER, H. Nutrição mineral da cana-deaçúcar (micronutrientes). In: ORLANDO FILHO, J., ed. Nutrição e adubação da cana-de-açúcar no Brasil. 2.ed. Piracicaba, PLANALSUCAR, 1983. p.103-122.

TASSO JUNIOR, L.C.; MARQUES, M.O.; CAMILOTTI, F. \& SILVA, T. Extração e exportação de macronutrientes em cinco variedades de cana-de-açúcar cultivadas na região centro-norte do Estado de São Paulo. STAB, 25:38-42, 2007.

THANGAVELU, S. \& CHIRANJIVI RAO, K. Calcium, magnesium and sulphur uptake by above ground parts in intergeneric hybrids. Sugar Tec., 6:25-33, 2004.

WOOD, A.W.; MUCHOW, R.C. \& ROBERTSON, M.J. Growth of sugarcane under higt input conditions in tropical Australia, III. Accumulation, partitioning and use of nitrogen. Field Crop. Res., 48:223-233, 1996. 